\title{
A Radon Slantlet Transforms Based OFDM System Design and Performance Simulation under Different Channel Conditions
}

\begin{abstract}
Abbas Hasan Kattoush
EE Department, Tafila Technical University, Tafila 66110, Jordan

Correspondence should be addressed to Abbas Hasan Kattoush, akattoush@yahoo.ca

Received 19 October 2011; Accepted 29 November 2011

Academic Editor: K. Teh

Copyright () 2012 Abbas Hasan Kattoush. This is an open access article distributed under the Creative Commons Attribution License, which permits unrestricted use, distribution, and reproduction in any medium, provided the original work is properly cited.

Due to its good orthogonality, slantlet transform (SLT) is used in orthogonal frequency division multiplexing (OFDM) systems to reduce intersymbol interference (ISI) and intercarrier interference (ICI). This eliminates the need for cyclic prefix (CP) and increases the spectral efficiency of the design. Finite Radon transform (FRAT) mapper has the ability to increase orthogonality of subcarriers, is nonsensitive to channel parameters variations, and has a small constellation energy compared with conventional fast-Fourier-transform- (FFT-) based OFDM. It is also able to work as a good interleaver, which significantly reduces the bit error rate (BER). In this paper both FRAT mapping technique and SLT modulator are implemented in a new design of an OFDM system. The new structure was tested and compared with conventional FFT-based OFDM, Radon transform-based OFDM, and SLT-based OFDM for additive white Gaussian noise (AWGN) channel, flat fading channel (FFC), and multipath selective fading channel (SFC). Simulation tests were generated for different channel parameters values. The obtained results showed that the proposed system has increased the spectral efficiency, reduced ISI and ICI, and improved BER performance compared with other systems.
\end{abstract}

\section{Introduction}

Orthogonal frequency division multiplexing system is one of the most promising technologies for current and future wireless communications. It is a form of multicarrier modulation technologies where data bits are encoded to multiple subcarriers, while being sent simultaneously [1]. Each subcarrier in an OFDM system is modulated in amplitude and phase by the data bits. Modulation techniques typically used are binary phase shift keying, quadrature phase shift keying (QPSK), quadrature amplitude modulation (QAM), 16-QAM, 64-QAM, and so forth. The process of combining different subcarriers to form a composite time-domain signal is achieved using FFT and inverse FFT (IFFT) operations [2].

The main problem in the design of a communications system over a wireless link is to deal with multipath fading, which causes a significant degradation in terms of both the reliability of the link and the data rate [3]. Multipath fading channels have a severe effect on the performance of wireless communication systems even those systems that exhibit efficient bandwidth, like OFDM. There is always a need for developments in the realization of these systems as well as efficient channel estimation and equalization methods to enable these systems to reach their maximum performance [4]. The OFDM receiver structure allows relatively straightforward signal processing to combat channel delay spreads, which was a prime motivation to use OFDM modulation methods in several standards [5-8].

In transmissions over a radio channel, the orthogonality of the signals is maintained only if the channel is flat and time-invariant and channels with a Doppler spread and the corresponding time variations corrupt the orthogonality of the OFDM subcarrier waveforms [9]. In a dispersive channel, self-interference occurs among successive symbols at the same subcarrier casing ISI, as well as among signals at different subcarriers casing ICI. For a time-invariant but frequency-selective channel, ICI, as well as ISI, can effectively be avoided by inserting a cyclic prefix before each block of parallel data symbols at the cost of power loss and bandwidth expansion [2].

Conventional OFDM/QAM systems are robust for multipath channels due to the cyclically prefixed guard interval 
that is inserted between consequent symbols to cancel ISI. However, this guard interval decreases the spectral efficiency of the OFDM system as the corresponding amount [10]. Thus, there have been approaches of wavelet-based OFDM that do not require the use of the guard interval [11-16]. It is found that OFDM based on Haar orthonormal wavelets is capable of reducing the ISI and ICI, which are caused by the loss in orthogonality between the carriers.

Recently, Selesnick [17] has constructed a new orthogonal discrete wavelet transform, the slantlet transform, with two zero moments and improved time localization [18]. SLT transform found important applications in signal and image processing; it has been successfully applied in image compression and denoising. Also, SLT found a new application in communications, it was used as a main building block in designing an improved spectral efficiency OFDM system where the SLT modulator replaces the FFT modulator.

The Radon transform (RT) was first introduced by Radon [19, 20] and the theory, basic aspects, and applications of this transform are studied in [21, 22] while the finite Radon transform (FRAT) was first studied by Beylkin [23]. RT is the underlying fundamental concept used for computerized tomography scanning, as well as for a wide range of other disciplines, including radar imaging, geophysical imaging, nondestructive testing, and medical imaging [21]. Recently, FRAT was proposed as a mapping technique in OFDM system [24-26].

In this paper the idea of one-dimensional serial Radon transform-based OFDM proposed in [25] is developed farther towards increasing spectral efficiency and reducing BER. Further performance gains and higher spectral efficiency were made by combining both FRAT and SLT in the design of OFDM system. Simulation results show that the proposed system has better performance than Fourier, Radon, and SLT transform-based OFDM under different channel conditions.

\section{Slantlet Transform-Based OFDM System}

The slantlet transform was first proposed by Selesnick [17]. It is an orthogonal discrete wavelet transform (DWT) with two zero moments and with improved time localization. It uses a special case of a class of bases described by Alpert et al. in [27], the construction of which relies on GramSchmidt orthogonalization. SLT is based on the principle of designing different filters for different scales unlike iterated filter-bank approaches for the DWT. Selesnick described the basis from a filter-bank viewpoint, gave explicit solutions for the filter coefficients, and described an efficient algorithm for the transform.

The usual iterated DWT filter-bank and its equivalent form are shown in Figure 1. The "slantlet" filter-bank is based on the equivalent structure that is occupied by different filters that are not products. With this extra degree of freedom obtained by giving up the product form, filters of shorter length are designed satisfying orthogonality and zero moment conditions.

For the two-channel case the Daubechies filter [28] is the shortest filter, which makes the filter-bank orthogonal and

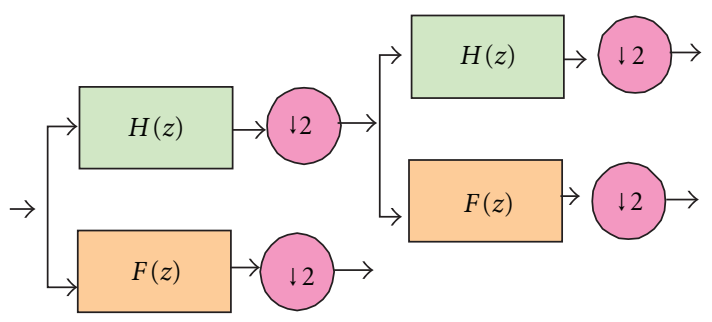

(a)

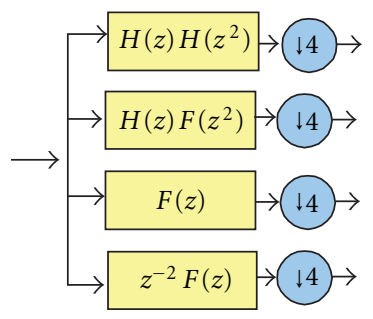

(b)

FIgure 1: Two-scale iterated filter-bank and its equivalent form using the DWT.

has $K$ zero moments. For $K=2$ zero moments, filters $H(z)$ and $F(z)$ are of length 4 . For this system, the iterated filters in Figure 1 are of length 10 and 4. Without the constraint that the filters are products, an orthogonal filter-bank with $K=2$ zero moments can be obtained where the filter lengths are 8 and 4, as shown in Figure 2. That is a reduction by two samples, which is a difference that grows with the number of stages. This reduction in length, while maintaining desirable orthogonality and moment properties, is possible because these filters are not constrained by the product form arising in the case of iterated filter-banks.

The filters coefficients used in the SLT filter-bank as derived in [17] are given by

$$
\begin{aligned}
G_{1}(z)= & \left(-\frac{\sqrt{10}}{20}-\frac{\sqrt{2}}{4}\right)+\left(\frac{3 \sqrt{10}}{20}+\frac{\sqrt{2}}{4}\right) z^{-1} \\
& +\left(-\frac{3 \sqrt{10}}{20}+\frac{\sqrt{2}}{4}\right) z^{-2}+\left(\frac{\sqrt{10}}{20}-\frac{\sqrt{2}}{4}\right) z^{-3} \\
F_{2}(z)= & \left(\frac{7 \sqrt{5}}{80}-\frac{3 \sqrt{55}}{80}\right)+\left(-\frac{\sqrt{5}}{80}-\frac{\sqrt{55}}{80}\right) z^{-1} \\
& +\left(-\frac{9 \sqrt{5}}{80}+\frac{\sqrt{55}}{80}\right) z^{-2}+\left(-\frac{17 \sqrt{5}}{80}+\frac{3 \sqrt{55}}{80}\right) z^{-3} \\
& +\left(\frac{7 \sqrt{5}}{80}+\frac{3 \sqrt{55}}{80}\right) z^{-4}+\left(\frac{9 \sqrt{5}}{80}+\frac{\sqrt{55}}{80}\right) z^{-5} \\
& +\left(\frac{\sqrt{5}}{80}-\frac{\sqrt{55}}{80}\right) z^{-6}+\left(-\frac{7 \sqrt{5}}{80}-\frac{3 \sqrt{55}}{80}\right) z^{-7}
\end{aligned}
$$




$$
\begin{aligned}
H_{2}(z)= & \left(\frac{1}{16}+\frac{\sqrt{11}}{16}\right)+\left(\frac{3}{16}+\frac{\sqrt{11}}{16}\right) z^{-1} \\
& +\left(\frac{5}{16}+\frac{\sqrt{11}}{16}\right) z^{-2}+\left(\frac{7}{16}+\frac{\sqrt{11}}{16}\right) z^{-3} \\
& +\left(\frac{7}{16}-\frac{\sqrt{11}}{16}\right) z^{-4}+\left(\frac{5}{16}-\frac{\sqrt{11}}{16}\right) z^{-5} \\
& +\left(\frac{3}{16}-\frac{\sqrt{11}}{16}\right) z^{-6}+\left(\frac{1}{16}-\frac{\sqrt{11}}{16}\right) z^{-7}
\end{aligned}
$$

Some characteristic features of the SLT filter-bank are being orthogonal and having two zero moments, and it also has octave-band characteristic. Each filter-bank has a scale dilation factor of two and provides a multiresolution decomposition. The slantlet filters are piecewise linear. Even though there is no tree structure for SLT, it can be efficiently implemented like an iterated DWT filter-bank [17]. Therefore, a computational complexity of the SLT is of the same order as that of the DWT.

The block diagram of the SLT-OFDM system is depicted in Figure 2. It is very similar to that of FFT-OFDM; the only differences are in the OFDM modulator-demodulator blocks. The processes of serial to parallel (S/P) conversion, signal demapping, and insertion of training sequence are the same as in the FFT-OFDM system. The SLT-based OFDM modulator consists of zero padding and inverse SLT (ISLT) blocks while the SLT-based OFDM demodulator consists of SLT and zero-pad removal blocks as shown in Figure 2. The main difference between FFT-OFDM and SLT-OFDM is that in SLT-OFDM there is no need for adding a cyclic prefix to OFDM symbols as shown in Figure 3; therefore, the spectral efficiency and the data rate of SLT-OFDM are better than those of FFT-OFDM.

\section{The Radon Mapping Technique}

Radon transform-based OFDM was recently proposed [24, $25]$; it was found that, as a result of applying FRAT, the bit error rate (BER) performance was improved significantly, especially in the existence of multipath fading channels. Also, it was found that Radon transform-based OFDM structure is less sensitive to channel parameters variation, like maximum delay, path gain, and maximum Doppler shift in selective fading channels, as compared with the standard OFDM structure.

In Radon transform-based OFDM system, FRAT mapping is used instead of QAM mapping $[25,26]$. The other processing parts of the system remain the same as in the conventional QAM OFDM system. It is known that FFT based OFDM obtain the required orthogonality between subcarriers from the suitability of IFFT algorithm [2]. Using FRAT mapping with the OFDM structure increases the orthogonality between subcarriers since FRAT computation uses one-dimensional (1D) IFFT algorithm. Also, FRAT is designed to increase the spectral efficiency of the OFDM system through increasing the bit per Hertz of the mapping.
Subcarriers are generated using $N$ points discrete Fourier transform (DFT), and guard interval (GI) inserted at the start of each symbol is used to reduce ISI.

The procedure steps of using the serial Radon transformbased OFDM mapping are as follows [25].

Step 1. Suppose $d(k)$ is the serial data stream to be transmitted using OFDM modulation scheme. Converting $d(k)$ from serial form to parallel form will construct a one-dimensional vector containing the data symbols to be transmitted:

$$
d(k)=\left(\begin{array}{lllll}
d_{0} & d_{1} & d_{2} & \cdots & d_{n}
\end{array}\right)^{T},
$$

where $k$ and $n$ are the time index and the vector length, respectively.

Step 2. Convert the data packet represented by the vector $d(k)$ from one-dimensional vector to a $p \times p$ twodimensional matrix $D(k)$, where $p$ should be a prime number according to the matrix resizing operation.

Step 3. Take the two-dimensional (2D) FFT of the matrix $D(k)$ to obtain the matrix, $F(r, s)$. For simplicity it will be labeled by $F$ :

$$
F(r, s)=\sum_{m=0}^{p-1} \sum_{n=0}^{p-1} D(m, n) e^{-j(2 \pi / p) r m} e^{-j(2 \pi / p) n s} .
$$

Step 4. Redistribute the elements of the matrix $\mathcal{F}$ according to the following optimum ordering algorithm first described in [29]. The optimum number of FRAT projections is one projection for each column, and the best ordering of the $2 \mathrm{D}$ FFT coefficients in these projections can be achieved if the normal vectors are determined as [29]

$$
\begin{gathered}
\left(a_{k}, b_{k}\right)=\arg \min \left|\left(C_{p}\left(a_{k}\right), C_{p}\left(b_{k}\right)\right)\right|, \\
\left(a_{k}, b_{k}\right) \in\left\{n u_{k}: 1 \leq n \leq p-1\right\}, \\
\text { st. } C_{p}\left(b_{k}\right) \geq 0 .
\end{gathered}
$$

Here, $C_{p}(x)$ denotes the centralized function of period $p ; C_{p}(x)=x-p \cdot$ round $(x / p)$. Hence, $\left\|\left(C_{p}\left(a_{k}\right), C_{p}\left(b_{k}\right)\right)\right\|$ represents the distance from the origin to the point $\left(a_{k}, b_{k}\right)$ on the Fourier plane. The constraint $C_{p}\left(b_{k}\right) \geq 0$ is imposed in order to remove the ambiguity in deciding between $(a, b)$ and $(-a,-b)$ as the normal vector for the projection. As a result the optimal normal vectors are restricted to having angles in $[0, \pi)$. So, the dimensions of the resultant matrix will be $p \times(p+1)$ and will be denoted by the symbol $\mathcal{F}_{\text {opt }}$.

Step 5. Take the 1D IFFT for each column of the matrix $\mathcal{F}_{\text {opt }}$ to obtain the matrix of Radon coefficients, $R$ :

$$
R=\frac{1}{p} \sum_{k=0}^{N-1} \mathcal{F}_{\mathrm{opt}} e^{j 2 \pi k n / p}
$$

Step 6. Construct the complex matrix $\bar{R}$ from the real matrix $R$ such that its dimensions will be $p \times(p+1) / 2$ according to

$$
\overline{r_{l, m}}=r_{i, j}+j r_{i, j+1}, \quad 0 \leq i \leq p, 0 \leq j \leq p,
$$




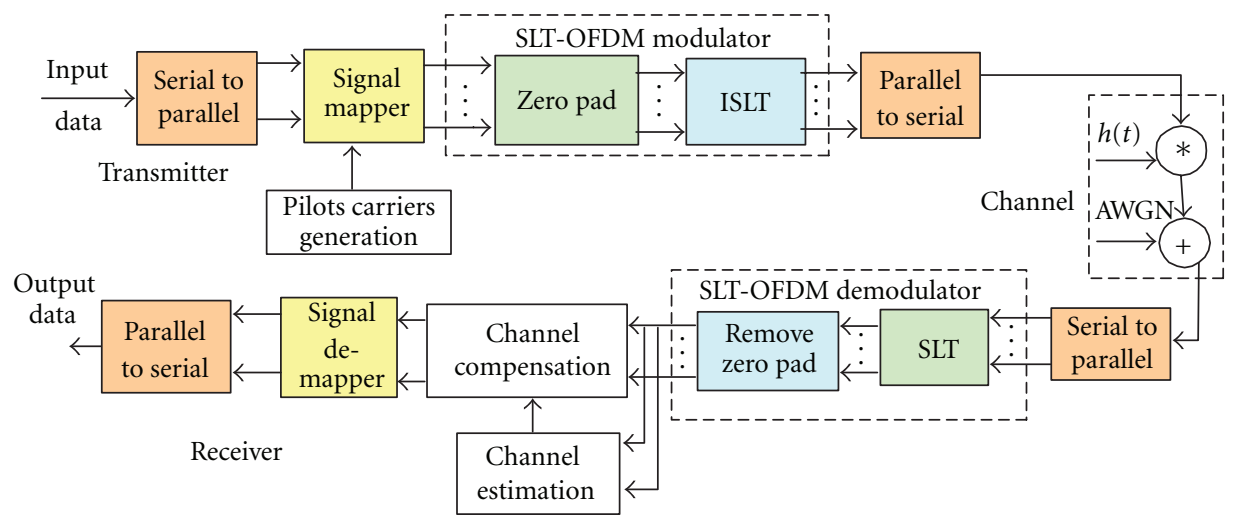

FIGURE 2: Block diagram of SLT-OFDM system.

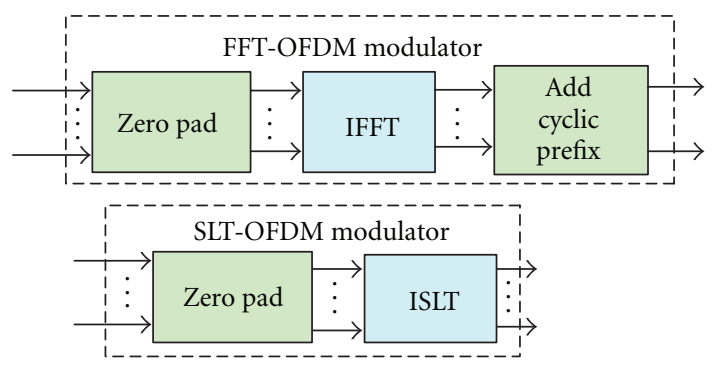

Figure 3: FFT-OFDM and SLT-OFDM modulators.

where $\overline{r_{l, m}}$ refers to the elements of the matrix $\bar{R}$, while $r_{i, j}$ refers to the elements of the matrix $R$. Matrixes $R$ and $\bar{R}$ are given by

$$
\begin{aligned}
& R=\left[\begin{array}{ccccc}
r_{1,1} & r_{1,2} & r_{1,3} & \cdots & r_{1, p+1} \\
r_{2,1} & r_{2,2} & r_{2,3} & \cdots & r_{2, p+1} \\
\vdots & \vdots & \vdots & \cdots & \vdots \\
\vdots & \vdots & \vdots & \cdots & \vdots \\
r_{p-1,1} & r_{p-1,2} & \cdots & \cdots & r_{p-1, p+1} \\
r_{p, 1} & r_{p, 2} & r_{p, 3} & \cdots & r_{p, p+1}
\end{array}\right] \\
& \bar{R}=\left[\begin{array}{cccc}
r_{1,1}+j r_{1,2} & r_{1,3}+j r_{1,4} & \cdots & r_{1, p}+j r_{1, p+1} \\
r_{2,1}+j r_{2,2} & r_{2,3}+j r_{2,4} & \cdots & r_{2, p}+j r_{2, p+1} \\
\vdots & \vdots & \cdots & \vdots \\
\vdots & \vdots & \cdots & \vdots \\
r_{p-1,1}+j r_{p-1,2} & \cdots & \cdots & r_{p-1, p}+j r_{p-1, p+1} \\
r_{p, 1}+j r_{p, 2} & \cdots & \cdots & r_{p, p}+j r_{p, p+1}
\end{array}\right] \text {. }
\end{aligned}
$$

Complex matrix construction is made for a purpose of increasing the bit per Hertz of mapping before resizing the mapped data.
Step 7. Resize the matrix $\bar{R}$ to a one-dimensional vector $r(k)$ of length $p \times(p+1) / 2$ :

$$
r(k)=\left(\begin{array}{lllll}
r_{0} & r_{1} & r_{2} & \cdots & r_{p(p+1) / 2}
\end{array}\right)^{T} .
$$

Step 8. Take the 1D IFFT for the vector $r(k)$ to obtain the subchannel modulation:

$$
s(k)=\frac{1}{p(p+1) / 2} \sum_{k=0}^{N_{C}-1} r(k) e^{j 2 \pi k n /(p(p+1) / 2)},
$$

where $N_{C}$ referes to number of carriers.

Step 9. Finally, convert the vector $s(k)$ to serial data symbols: $s_{0}, s_{1}, s_{2}, \ldots, s_{n}$.

\section{Proposed System for Radon-SLT-Based OFDM Transceiver}

Due to good orthogonality of both SLT and FRAT, which reduce ISI and ICI, in the proposed system there is no need of using cyclic prefix (CP). The block diagram of the proposed Radon-SLT-based OFDM system is depicted in Figure 4 and the ISLT modulator and SLT demodulator are also shown in the same figure.

The processes of serial to parallel conversion, signal demapping, and insertion of training sequence are the same as in the system of FFT-OFDM. Also, the zeros are added as in the FFT-based case and for the same reasons. After that the ISLT is applied to the signal. The main and important difference between FFT-based OFDM and SLT-based OFDM is that in SLT-based OFDM the cyclic prefix is not added to OFDM symbols. Therefore, the data rates in SLT-based OFDM are higher than those of the FFT-based OFDM. At the receiver, the zeros padded at the transmitter are removed, and the other operations of channel estimation, channel compensation, signal demapping, and parallel to serial (P/S) conversion are performed in the same manner as in FFTbased OFDM.

In the conventional OFDM system, the length of input data frame is 60 symbols, and after S/P conversion and QAM mapping the length becomes 30 symbols. Zero padding 


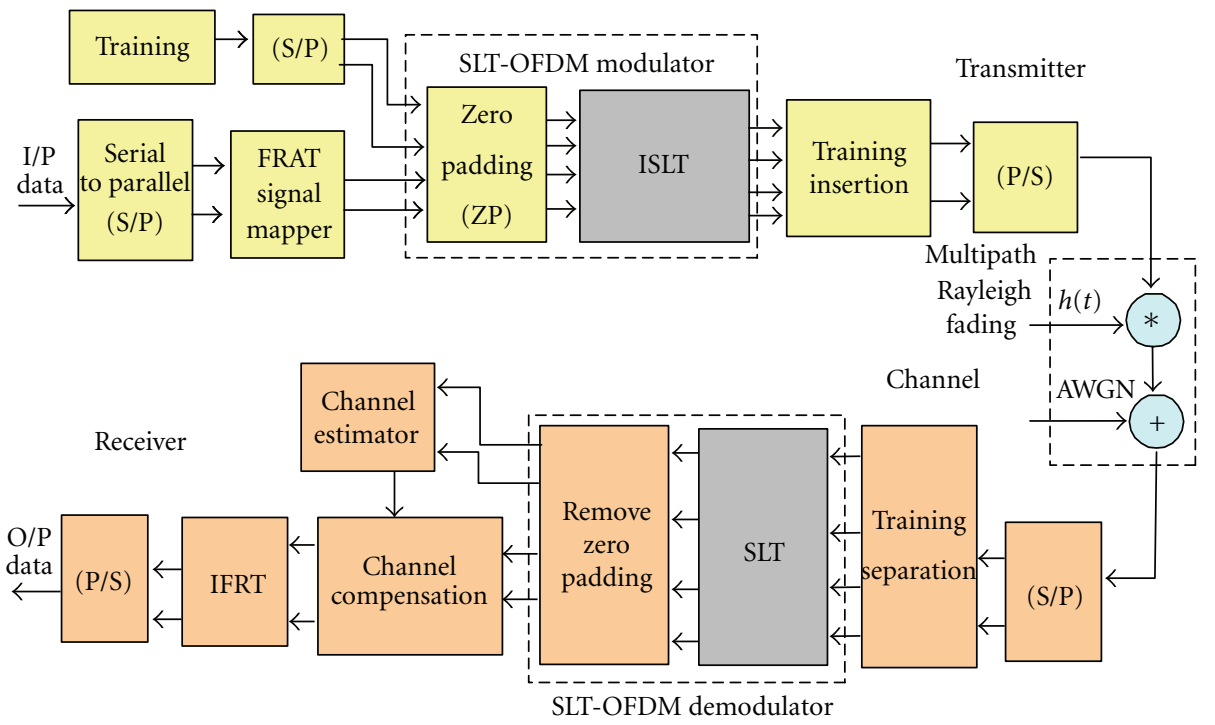

Figure 4: Block diagram of FRAT-SLT-based OFDM system.

operation makes the length 64 symbols, which are the input to IFFT (subcarrier modulation). After adding CP (usually $40 \%$ of the length of the frame), the frame length becomes 90 symbols. Since OFDM operations applied to the training symbols are the same as those applied to the transmitted data (except the mapping operation), the length of the training symbols is also 90 symbols. The training and data frames are transmitted as one frame starts with training, so the length of transmitted frame is 180 symbols [30]. In the proposed system, the length of the input data frame must be $(p \times p)$, where $p$ is a prime number. The closest number to 60 is $(7 \times 7)$, which makes the frame length 49 symbols. This is because the input of FRAT must be a two-dimensional matrix with size $(p \times p)$.

\section{Simulation Results of Proposed System}

Four types of OFDM systems were simulated: FFT-OFDM, Radon-OFDM, SLT-OFDM, and proposed Radon-SLTbased OFDM systems using MATLAB version 7. The BER performances of the four systems were found for different channel models: AWGN channel, flat fading channel, and selective fading channel. System parameters used through the simulations are $T_{S}=0.1 \mu \mathrm{sec}$, FRAT window: 7 by 7 , and DWT bins $N=64$.

5.1. Performance of Proposed OFDM System in AWGN Channel. Figure 5 shows the results of simulation of the proposed system compared with other systems in AWGN channel. It is clearly seen that FRAT-SLT-based OFDM has better performance than the other three systems: FFTOFDM, SLT-OFDM, and FRAT-OFDM. This is due to the high orthogonality of the proposed system. To have BER = $10^{-4}$, FFT-OFDM requires $28 \mathrm{~dB}$, FRAT-OFDM requires $25.5 \mathrm{~dB}$, SLT-OFDM requires $21.5 \mathrm{~dB}$, and FRAT-SLT-based OFDM requires $17 \mathrm{~dB}$. And to have $\mathrm{BER}=10^{-5}$, FFT-OFDM requires $31.5 \mathrm{~dB}$, FRAT-OFDM requires $28 \mathrm{~dB}$, SLT-OFDM

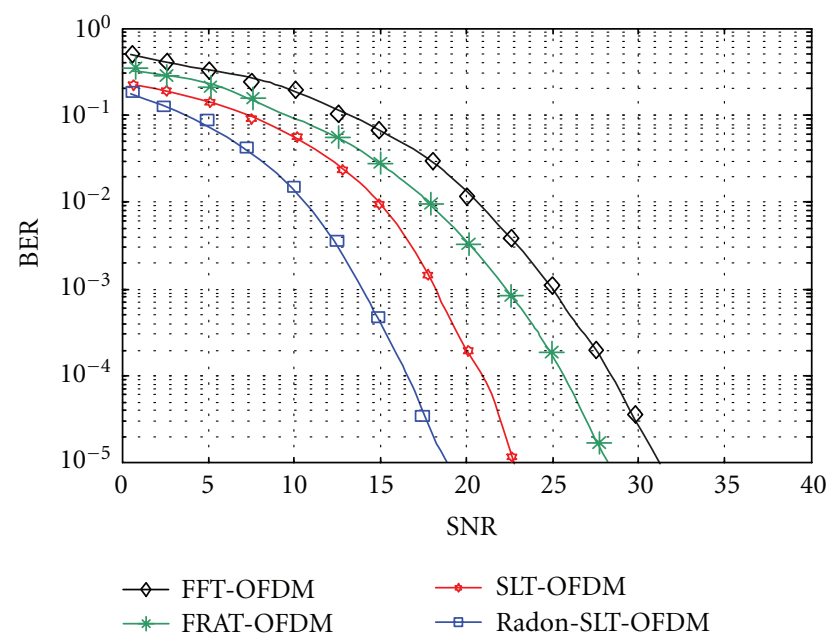

FIGURE 5: BER performance of FRAT-SLT-based OFDM in AWGN channel.

requires $23 \mathrm{~dB}$, and FRAT-SLT-based OFDM requires $19 \mathrm{~dB}$. From the results it can be noted that the proposed system has $12 \mathrm{~dB}$ advantage over FFT-OFDM, 9.5 dB over FRAT-OFDM, and $5 \mathrm{~dB}$ over SLT-OFDM.

5.2. Performance of Proposed OFDM System in Flat Fading Channel with AWGN. In this channel, all signal frequency components are affected by a constant attenuation and linear phase distortion, in addition to an AWGN. The channel was selected to be multipath and Rayleigh distributed. Doppler frequency used in simulation is calculated as follows: $c=$ $300 \times 10^{6} \mathrm{~m} / \mathrm{sec}$, in GSM system $f_{c}=900 \mathrm{MHz}$ so,

$$
\begin{gathered}
f_{d}=f_{c} \times \frac{v}{c}=900 \times 10^{6} \times \frac{1}{\mathrm{sec}} \times \frac{v}{300 \times 10^{8} \mathrm{~m} / \mathrm{sec}}, \\
f_{d}=\frac{3}{(1 \mathrm{~m})} \times v .
\end{gathered}
$$




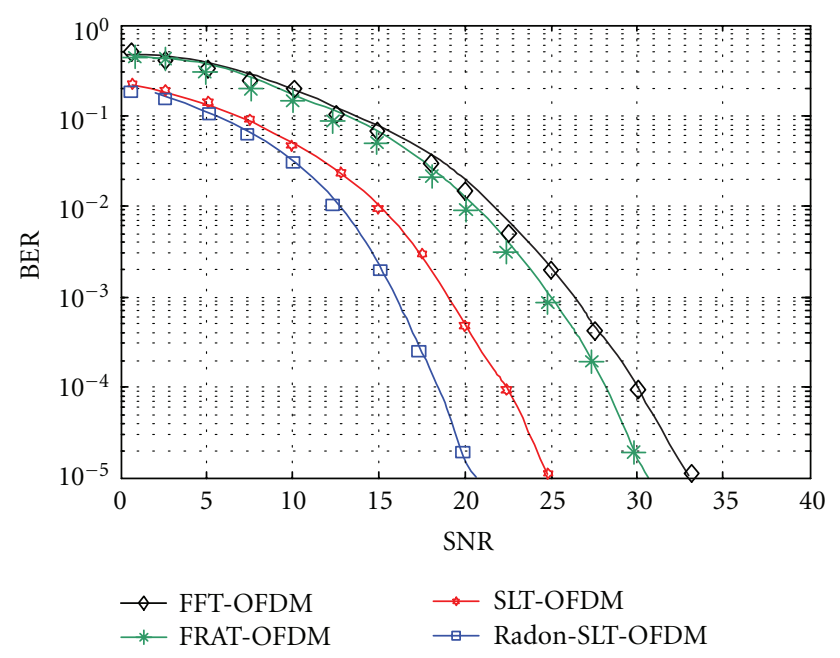

FIGURE 6: BER performance of FRAT-SLT-OFDM in FFC at Doppler frequency $4 \mathrm{~Hz}$.

The Doppler frequency used is that corresponding to a walking speed $(4.8 \mathrm{~km} /$ hour $)$, and it has a value $f_{d}=$ $(3 / 1 \mathrm{~m}) \times(4.8 \times 1000 \mathrm{~m} / 3600 \mathrm{sec})=4 \mathrm{~Hz}$.

The results of simulations for $4 \mathrm{~Hz}$ Doppler frequency are shown in Figure 6. From Figure 6 it can be seen that to have $\mathrm{BER}=10^{-5}$, FFT-OFDM requires $33 \mathrm{~dB}$, FRAT-OFDM requires $31 \mathrm{~dB}$, SLT-OFDM requires $25 \mathrm{~dB}$, and FRAT-SLTbased OFDM requires $20.5 \mathrm{~dB}$. So the proposed system offers $12.5 \mathrm{~dB}$ SNR improvement compared with FFTOFDM, $10.5 \mathrm{~dB}$ compared with FRAT-OFDM, and $4.5 \mathrm{~dB}$ compared with SLT-OFDM for this channel model. Other Doppler-Shift frequencies were used for proposed system simulation over the flat fading Rayleigh channel; the values used are $80 \mathrm{~Hz}$ corresponding to car speed ( $96 \mathrm{~km} /$ hour), $300 \mathrm{~Hz}$ corresponding to helicopter speed ( $360 \mathrm{~km} / \mathrm{hour}$ ), and $500 \mathrm{~Hz}$ corresponding to airplane speed $(600 \mathrm{~km} / \mathrm{hour})$, and the same results were obtained for these frequencies. The reason for best performance results of FRAT-SLT-based OFDM is the good orthogonality of Radon transform and the excellent orthogonality of SLT.

\subsection{BER Performance of Proposed OFDM System in SFC with} $A W G N$. In this section, the channel model is assumed to be selective fading channel. A second ray Raleigh-distributed multipath fading channel is assumed, where the parameters of the multipaths channel are path gain equal to $-8 \mathrm{~dB}$ and path delay $\tau_{\max }=0.1 \mu \mathrm{sec}$.

The BER performance of the proposed system and the other OFDM systems over a selective fading channel with Doppler frequency of $4 \mathrm{~Hz}$ is shown in Figure 7. It can be seen that to have BER $=10^{-5}$, FFT-OFDM requires $37.5 \mathrm{~dB}$, FRAT-OFDM requires $35.5 \mathrm{~dB}$, SLT-OFDM requires $29 \mathrm{~dB}$, and FRAT-SLT-based OFDM requires $22.5 \mathrm{~dB}$. So the proposed system offers great SNR improvement compared with FFT-OFDM, FRAT-OFDM, and SLT-OFDM for this channel model.

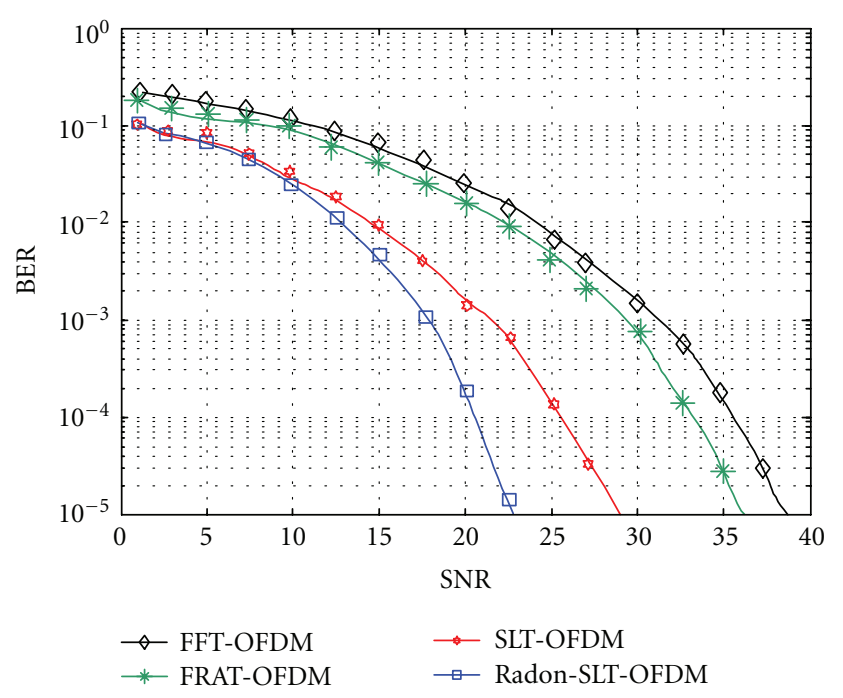

FIGURE 7: BER performance of FRAT-SLT-OFDM in SFC at Doppler frequency $4 \mathrm{~Hz}$.

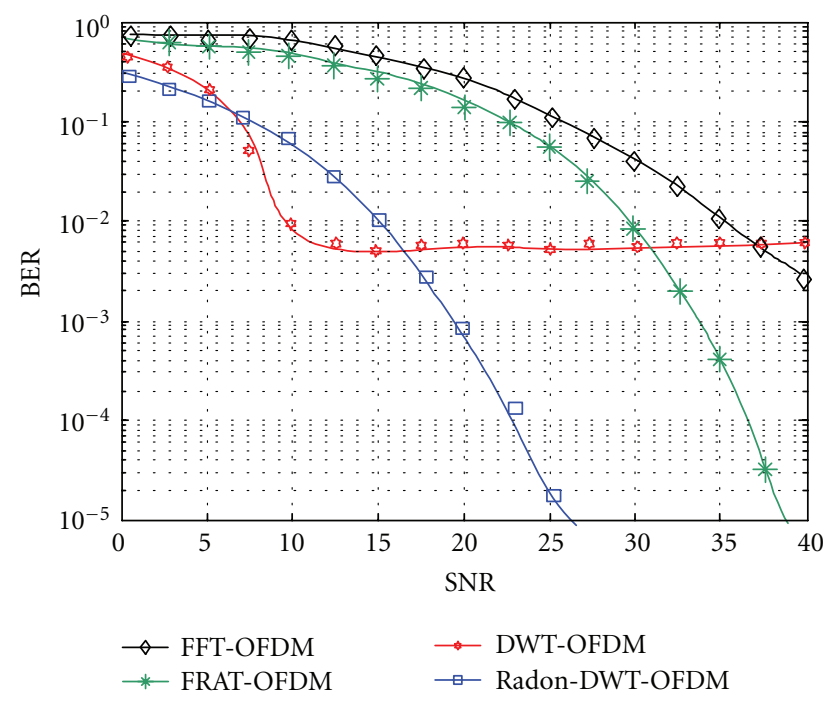

FIGURE 8: FRAT-SLT-OFDM BER performance in SFC at Doppler frequency $300 \mathrm{~Hz}$.

The same performance characteristics of the systems over selective fading channel with Doppler frequencies $80 \mathrm{~Hz}$, $300 \mathrm{~Hz}$, and $500 \mathrm{~Hz}$ were simulated. Figure 8 shows the BER performance of FRAT-SLT-based OFDM in selective fading channel with Doppler frequency of $300 \mathrm{~Hz}$. From Figure 8, it is clearly seen that FFT-based OFDM needs more than $40 \mathrm{~dB}$ of SNR to have BER $=10^{-4}$, while FRAT-based OFDM needs around $39 \mathrm{~dB}$ of SNR to reach BER $=10^{-5}$, SLT-based OFDM BER performance does not exceed 0.002425 with increasing SNR, whereas the proposed FRAT-SLT based OFDM has much better performance than the other three systems, and it reaches $\mathrm{BER}=10^{-5}$ at $\mathrm{SNR}=26.5 \mathrm{~dB}$.

Figure 9 shows the BER performance of FRAT-SLT-based OFDM in selective fading channel with Doppler frequency of $500 \mathrm{~Hz}$. From Figure 9, the following conclusion can 


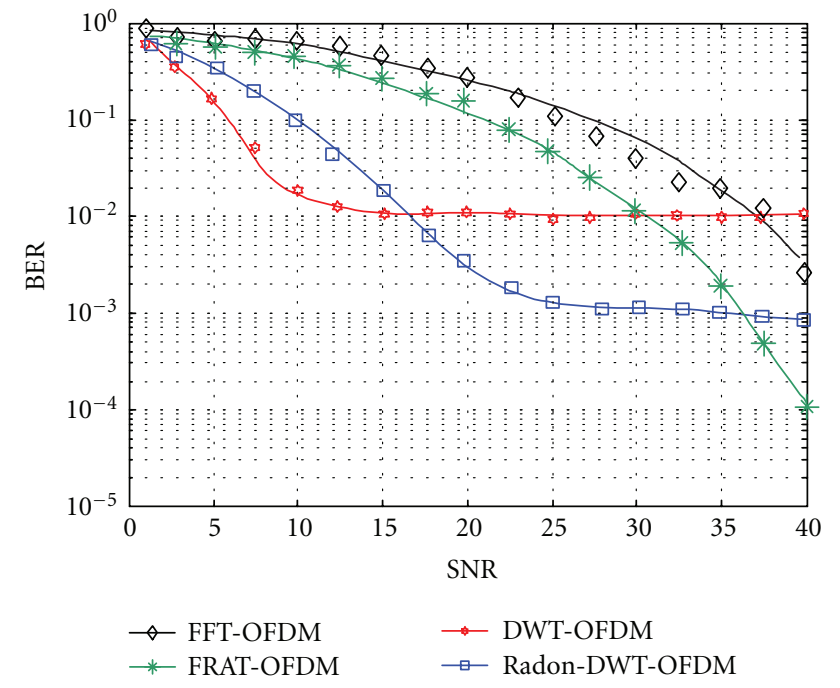

FIGURE 9: BER performance of FRAT-SLT-OFDM in SFC at Doppler frequency $500 \mathrm{~Hz}$.

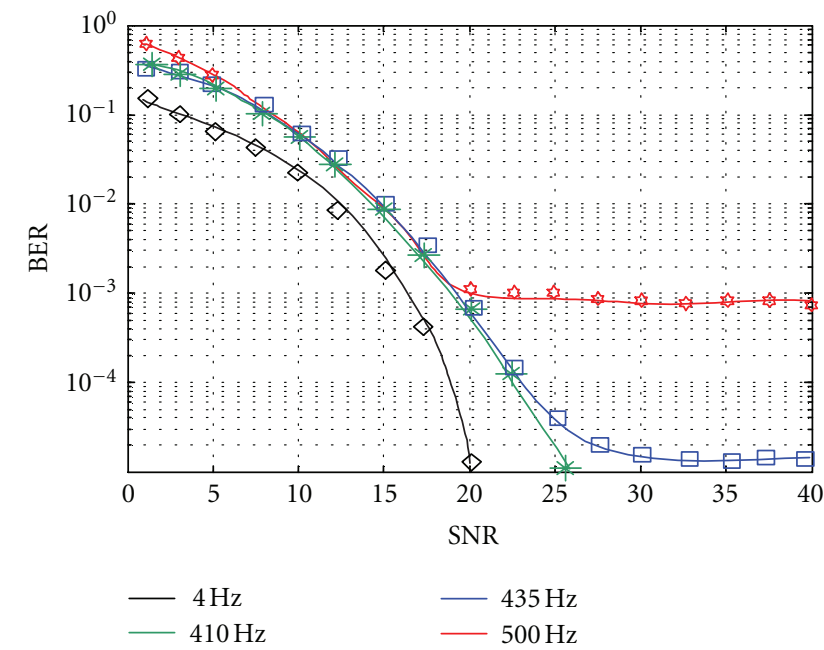

FIGURE 10: FRAT-SLT-OFDM BER performance in SFC at different Doppler frequencies.

be stated: when Doppler frequency exceeds $500 \mathrm{~Hz}$, the proposed system suffers from the same problem that SLTbased OFDM system suffers from, and the performance of the proposed system does not increase with increasing SNR when the Doppler frequency exceeds $500 \mathrm{~Hz}$. It is seen that OFDM systems are very sensitive systems to the variation of Doppler frequency in selective fading channel.

The effect of Doppler frequency value on BER performance for the proposed system is provided in Figure 10. It can be seen from Figure 10 that the critical value of Doppler frequency for the proposed system is around $435 \mathrm{~Hz}$.

\section{Conclusions}

In this paper a novel OFDM generation method is proposed, simulated, and tested. The proposed system uses RadonSLT mapping instead of QAM mapping, which increases the orthogonality. The optimal ordering (best direction) in the Radon mapper can be considered as a good interleaver, which serves in error spreading. In the proposed system there is no need for using CP because of excellent orthogonality offered by FRAT and SLT, which in its order reduces the system complexity, increases the transmission rate, and increases spectral efficiency. Simulation results of the proposed RadonSLT-based OFDM show a very good SNR gain improvement and a BER performance as compared with SLT-OFDM, FRAT-OFDM, and FFT-OFDM in an AWGN, a flat fading, and a selective fading channels. It offers more than $15 \mathrm{~dB}$ SNR improvement compared with FFT-OFDM for selective fading channel at Doppler frequency $4 \mathrm{~Hz}$. From the simulation results, it can be seen that the proposed Radon-SLTbased OFDM has the smallest sensitivity to variations of the channel parameters.

\section{References}

[1] N. Al-Dhahir, "Optimum finite-length equalization for multicamer transceivers," IEEE Transactions on Communications, vol. 44, no. 1, pp. 56-64, 1996.

[2] S. B. Weinstein and P. M. Ebert, "Data transmission by frequency-division multiplexing using the discrete Fourier transform," IEEE Transactions on Communications, vol. 19, no. 5, pp. 628-634, 1971.

[3] N. H. Tran, H. H. Nguyen, and T. Le-Ngoc, "Bit-interleaved coded OFDM with signal space diversity: subcarrier grouping and rotation matrix design," IEEE Transactions on Signal Processing, vol. 55, no. 3, pp. 1137-1149, 2007.

[4] W. G. Jeon, K. H. Chang, and Y. S. Cho, "An equalization technique for orthogonal frequency-division multiplexing systems in time-variant multipath channels," IEEE Transactions on Communications, vol. 47, no. 1, pp. 27-32, 1999.

[5] R. V. Nee and R. Prasad, OFDM for Wireless Multimedia Communications, Artech-House, London, UK, 2000.

[6] I. Koffman and V. Roman, "Broadband wireless access solutions based on OFDM access in IEEE 802.16," IEEE Communications Magazine, vol. 40, no. 4, pp. 96-103, 2002.

[7] R. Prasad, OFDM for Wireless Communications Systems, Artech-House, 2004.

[8] I. Lee, J. S. Chow, and J. M. Cioffi, "Performance evaluation of a fast computation algorithm for the DMT in high speed subscriber loop," IEEE Journal on Selected Areas in Communications, vol. 13, no. 9, pp. 1564-1570, 1995.

[9] L. J. Cimini Jr., "Analysis and simulation of a digital mobile channel using orthogonal frequency division multiplexing," IEEE Transactions on Communications, vol. 33, no. 7, pp. 665$675,1985$.

[10] S. Kang and K. Chang, "A novel channel estimation scheme for OFDM/OQAM-IOTA system," ETRI Journal, vol. 29, no. 4, pp. 430-436, 2007.

[11] A. R. Lindsey, "Wavelet packet modulation for orthogonally multiplexed communication," IEEE Transactions on Signal Processing, vol. 45, no. 5, pp. 1336-1339, 1997.

[12] S. Mallat, A Wavelet Tour of Signal Processing, Academic Press, New York, NY, USA, 2nd edition, 1999.

[13] IEEE Std., IEEE Proposal for 802.16.3, RM Wavelet Based (WOFDM) PHY Proposal for 802.16.3, Rainmaker Technologies, Inc., 2001. 
[14] X. D. Zhang, P. P. Xu, G. A. Zhang, and G. G. Bi, "Study on complex wavelet packet based OFDM modulation (CWPOFDM)," Tien Tzu Hsueh Pao/Acta Electronica Sinica, vol. 30, no. 4, pp. 477-479, 2002.

[15] H. Zhang, D. Yuan, and M. Pätzold, "Novel study on PAPRs reduction in wavelet-based multicarrier modulation systems," Digital Signal Processing, vol. 17, no. 1, pp. 272-279, 2007.

[16] A. H. Kattoush, W. A. Mahmoud, and S. Nihad, "The performance of multiwavelets based OFDM system under different channel conditions," Digital Signal Processing, vol. 20, no. 2, pp. 472-482, 2010.

[17] I. W. Selesnick, "The slantlet transform," IEEE Transactions on Signal Processing, vol. 47, no. 5, pp. 1304-1313, 1999.

[18] E. R. Dougherty, J. T. Astola, and K. O. Egiazarian, "The fast parametric slantlet transform with applications," in Image Processing: Algorithms and Systems III, vol. 5298 of Proceedings of SPIE, San Jose, Calif, USA, January 2004.

[19] J. Radon, "Über die Bestimmung von Funktionen durch ihre Integralwerte längs gewisser Mannigfaltigkeiten. Berichte über die Verhandlungen der Königlich Sächisischen Gesellshaft der Wissenschaften zu Luipzig," Mathematisch-Physikalische Klasse, vol. 69, pp. 262-277, 1917.

[20] J. Radon, "On the determination of functions from their integral values along certain manifolds," IEEE Transactions on Image Processing, vol. 5, pp. 170-176, 1986.

[21] S. R. Deans, The Radon Transform and Some of Its Applications, John Wiley \& Sons, New York, NY, USA, 1983.

[22] E. D. Bolker, “The finite radon transform," Integral Geometry, Contemporary Mathematics, vol. 63, pp. 27-50, 1987.

[23] G. Beylkin, "Discrete radon transforms," IEEE Transactions on Acoustics, Speech, and Signal Processing, vol. 35, no. 2, pp. 162172, 1987.

[24] W. Al-Jawhar, A. H. Kattoush, S. M. Abbas, and A. T. Shaheen, "A high performance parallel Radon based OFDM transceiver design and simulation," Digital Signal Processing, vol. 18, no. 6, pp. 907-918, 2008.

[25] A. H. Kattoush, W. A. Mahmoud, A. Shaheen, and A. Ghodayyah, "The performance of proposed one dimensional serial radon based OFDM system under different channel conditions," The International Journal of Computers, Systems and Signals, vol. 9, no. 2, pp. 3-16, 2008.

[26] A. H. Kattoush, W. A. M. Al-Jawher, S. M. Abbas, and A. T. Shaheen, "A n-radon based OFDM trasceivers design and performance simulation over different channel models," Wireless Personal Communications, vol. 58, no. 4, pp. 695-711, 2009.

[27] B. Alpert, G. Beylkin, R. Coifman, and V. Rokhlin, "Waveletlike bases for the fast solution of second-kind integral equations," SIAM Journal on Scientific Computing, vol. 14, pp. 159184, 1993.

[28] I. Daubechies, Ten Lectures on Wavelets, SIAM, Philadelphia, Pa, USA, 1992.

[29] M. N. Do and M. Vetterli, "The finite ridgelet transform for image representation," IEEE Transactions on Image Processing, vol. 12, no. 1, pp. 16-28, 2003.

[30] J. G. Proakis, Digital Communications, McGraw-Hill, 4ht edition, 2001. 

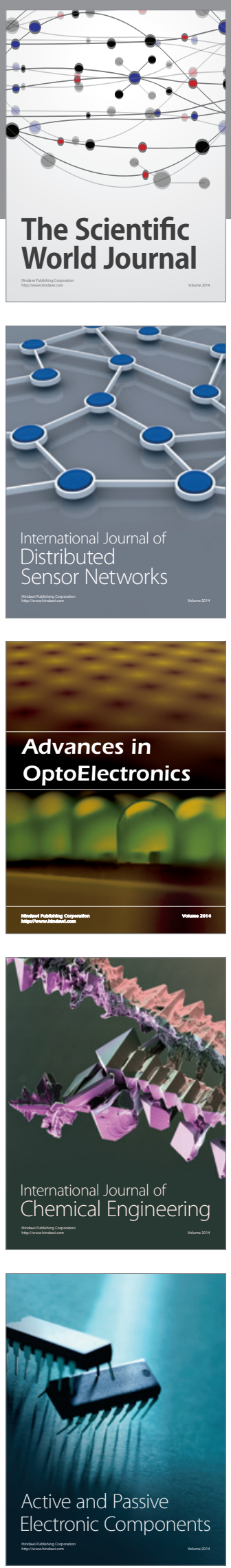
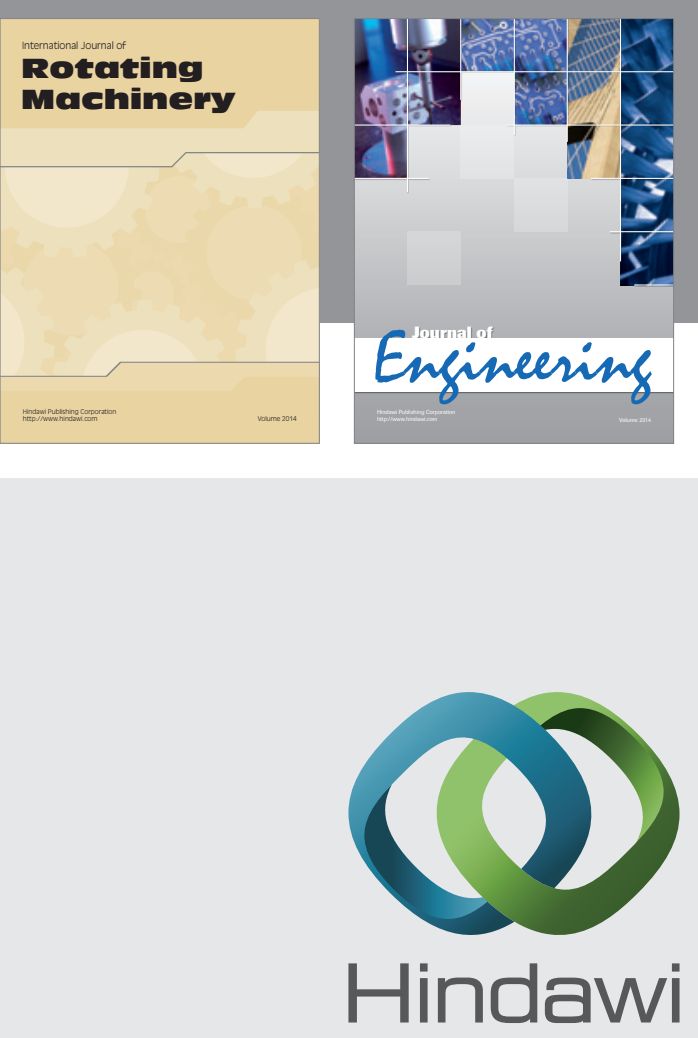

Submit your manuscripts at

http://www.hindawi.com
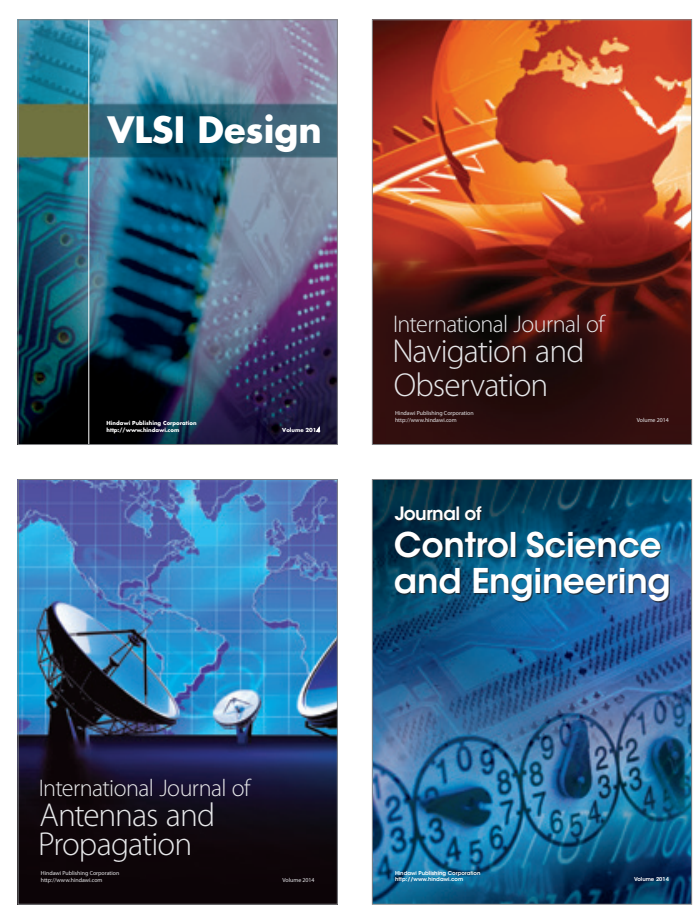
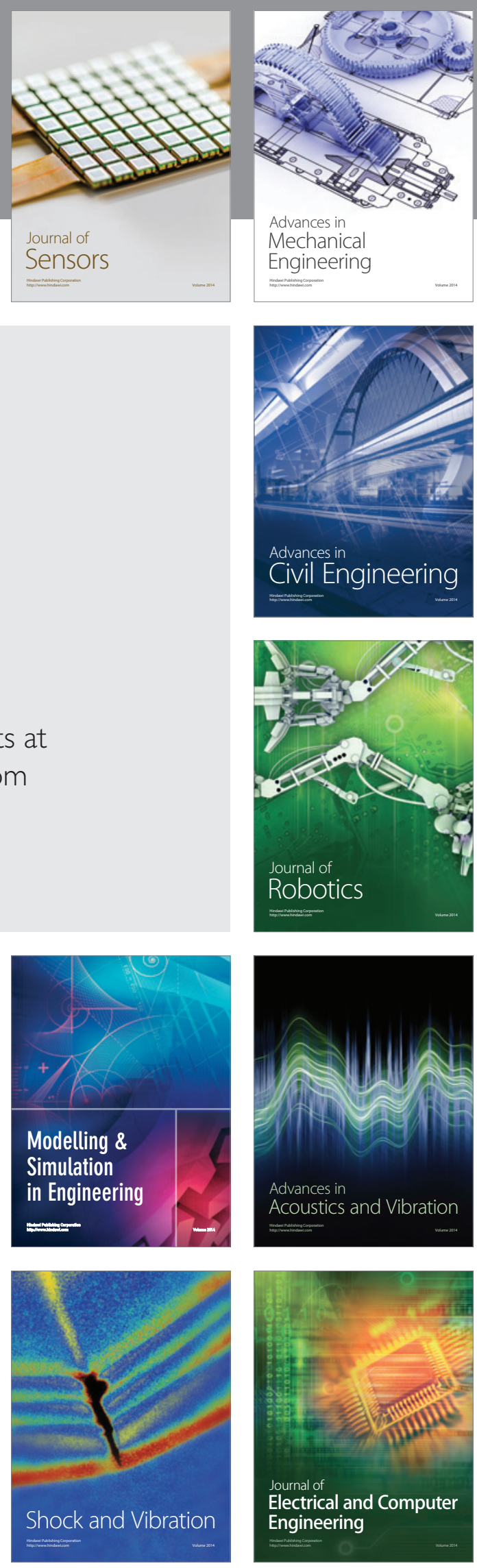